\title{
The Randomised Intervention Treatment of Angina (RITA) Trial protocol: a long term study of coronary angioplasty and coronary artery bypass surgery in patients with angina
}

\author{
R A HENDERSON, for the Randomised Intervention Treatment of Angina Trial
}

SUMMARY The Randomised Intervention Treatment of Angina (RITA) Trial is a prospective, randomised study to compare the short term and long term effects of percutaneous transluminal coronary angioplasty and coronary artery bypass surgery. During the study a register of patients undergoing coronary arteriography at the fourteen participating centres is being maintained to assess the overall context of patient recruitment. Patients with arteriographically proven coronary artery disease are considered for the trial if the participating cardiologist and surgeon agree that equivalent revascularisation could be achieved by either treatment method. Patients who satisfy the trial entry criteria are randomised to treatment by coronary angioplasty or coronary artery bypass surgery, with prospective stratification into groups with one, two, or three treatment vessels. Randomisation implies an intention to treat the patient by the assigned procedure and the analysis of long term results will include all randomised cases. The trial will recruit at least 1000 patients who will be followed for five years. The major trial end points include death, new myocardial infarction, and new coronary angioplasty or coronary artery bypass procedures. Other outcome measures include symptom and employment status, quality of life, exercise tolerance, and left ventricular function.

For many patients with coronary artery disease percutaneous transluminal coronary angioplasty and coronary artery bypass surgery offer alternative methods of improving myocardial blood supply. The long term effects of coronary artery surgery have been assessed in three randomised clinical trials, ${ }^{1-3}$ but information about the effects of coronary angioplasty is limited to registry studies. ${ }^{45}$

During 1986 and 1987 a small feasibility study examined the possibility of conducting a major randomised clinical trial to compare the effects of coronary angioplasty and coronary artery surgery. The feasibility study assessed whether patients and doctors would accept randomisation between such widely different treatments, and contributed to the

Requests for reprints to Dr R A Henderson, Department of Cardiology, Guy's Hospital, St Thomas Street, London SE1 9RT.

^See Appendix.

Accepted for publication 20 June 1989 development of a protocol for a large multicentre investigation. This protocol evolved into the Randomised Intervention Treatment of Angina (RITA) Trial, and a steering committee of interested and experienced cardiologists and surgeons was formed. Potential participating centres were invited to join the Randomised Intervention Treatment of Angina Trial and by November 1987 the trial protocol had been finalised. The Department of Health, the British Heart Foundation, and the British Cardiac Society agreed to support the Randomised Intervention Treatment of Angina Trial, and the first patient was randomised in March 1988. By July 1988 fourteen participating centres were entering patients into the trial.

\section{Trial objectives and design}

The Randomised Intervention Treatment of Angina Trial is a prospective, randomised, parallel group study to compare the short term and long term effects 
of percutaneous transluminal coronary angioplasty and coronary artery bypass surgery. Patients with arteriographically proven coronary artery disease are considered for the study if in the opinion of the participating cardiologist and cardiothoracic surgeon equivalent revascularisation could be achieved by either intervention procedure. All randomised patients will have detailed follow up for at least five years, and will be included in the analysis of long term results irrespective of the eventual treatment. The primary end points will be death, new myocardial infarction, and repeat coronary angioplasty or bypass surgery. An assessment will also be made of quality of life, exercise tolerance, and left ventricular function.

\section{Coronary arteriogram register}

To assess the relevance of the trial to clinical practice a register of all patients undergoing coronary arteriography at each participating centre is being maintained during the period of patient recruitment. The register is designed to encourage recruitment and to determine the reasons for excluding patients from the trial. The register is recording around 1000 patients per month and will provide independent information about the practice of coronary arteriography in the participating centres, which are sited throughout the United Kingdom.

\section{Patient eligibility}

Any patient, irrespective of sex or age, who has arteriographically proven coronary artery disease may be considered for the trial. Treatment by coronary angioplasty or coronary artery surgery must be considered necessary on clinical grounds, although it is not essential for patients to have angina if intervention is considered appropriate for reasons other than symptom relief. Patients with unstable angina, recent thrombolytic treatment, or poor left ventricular function may be included in the study as they form a large part of the routine workload of cardiac units. Patients with left main stem disease, previous coronary angioplasty or coronary bypass surgery, haemodynamically significant valve disease, or a systemic disease that will limit long term prognosis are all excluded from the study.

\section{Patient selection}

The coronary arteriograms of patients who may be eligible for the trial are reviewed by the participating cardiologist and cardiothoracic surgeon at each centre. The surgeon must be prepared to accept the patient for coronary artery surgery and the cardiolo- gist must consider that the patient is suitable foe coronary angioplasty. Before a patient can be ran domised the investigators must jointly identify the major epicardial vessels that require treatment and must agree that equivalent revascularisation could be achieved by either treatment method. Thus the surgeon must prospectively state which vessels $h \oplus$ intends to graft if the patient is randomised to surger and the cardiologist must be prepared to attempe? dilatation of all important stenoses in the same vessels. Coronary stenoses are regarded as important if there is a reduction in luminal diameter of at leas\$ $70 \%$ in one angiographic view or a $50 \%$ reduction in two views.

The trial does not require that all diseased vesselis are treated, but the intention to achieve equivalenis revascularisation by either treatment method may include totally occluded vessels. For instance, if $\overrightarrow{8}$ surgeon would graft the distal branches of a prox imally occluded artery the cardiologist must be prepared to attempt to reopen the occlusion bb coronary angioplasty. It is not necessary for angio plasty to be attempted in a vessel with subclinicap lesions even if the surgeon would graft the vessep during a surgical procedure.

\section{Stratification into groups with one, two, or three treatment vessels}

Patients are prospectively stratified into groups witt one, two, or three vessels requiring treatment patients with more than three treatment vessels ar\& ineligible for randomisation. All treatment vessels must be judged to supply $20 \%$ or more of the lef? ventricular myocardium and have at least one sig 5 nificant stenosis. A large side branch may qualify as separate treatment vessel, but small diseased sidè branches are not separate treatment vessels even i they would be grafted or dilated.

\section{Randomisation}

Randomisation is carried out via a dedicated tele phone line at the coronary care unit of the Royal Freo Hospital, London. Patients are randomly assigned to treatment by coronary angioplasty or coronary bypass surgery, with prospective stratification b⿸丆 centre and by one, two, or three treatment vesselso The date of randomisation marks the patient's entry to the trial, and the assigned intervention procedure్ should be carried out as soon as possible.

\section{Intervention procedure}

Before joining the trial each participating centre was required to be performing substantial numbers 
coronary angioplasty procedures, including multivessel dilatation, with a high success rate and low mortality. During the trial angioplasty and surgery will only be performed by fully trained operators. Because the trial will reflect contemporary cardiac practice no attempt has been made to standardise operative methods between centres.

In patients randomised to multivessel angioplasty some treatment vessels may not be successfully dilated at the first intervention procedure. Although the aim of intervention is to achieve equivalent revascularisation the decision to subject such patients to additional intervention procedures is to be based on clinical grounds.

\section{Data collection}

All data are recorded on specially prepared forms that are forwarded to the statistical centre and entered into a computerised data base. Baseline information recorded on all patients includes demographic data, cardiovascular risk factors, employment state, and physical activity level. Angina grade is assessed by the Canadian Cardiovascular Society Classification. ${ }^{6}$ Quality of life is measured by the Nottingham Health Profile, which is a standardised questionnaire designed to measure an individual's perceived health. ${ }^{7}$ Symptom limited exercise treadmill tests are performed according to the modified Bruce protocol, ${ }^{8}$ unless the test is contraindicated because of unstable angina or intercurrent illness. Left ventricular function is assessed by gated radionuclide left ventriculography, and although the method may vary between centres radionuclide scans in individual patients will be repeated at the same centre by the same method. The pre-intervention coronary arteriogram findings are documented and the treatment vessels are prospectively identified.

During follow up patients are reviewed one, six, and twelve months after the intervention procedure and then two, three, four, and five years after the date of randomisation. In addition, patients are contacted every three months to confirm vital state and establish the occurrence of new cardiovascular events.

\section{Trial events}

The major events to be recorded during the trial include death, myocardial infarction, and new angioplasty or bypass graft procedures. All deaths and potential new myocardial infarcts will be independently assessed by a central review committee who are not aware of the patient's assigned treatment. Although the cause of death will be independently established in each case, the main comparative results will be analysed by mortality from all causes.

In addition to these major trial events a range of other outcome measures are recorded. At each follow up assessment, employment and symptom status and performance on an exercise treadmill are documented. The radionuclide ejection fraction is measured one and six months after intervention, and the Nottingham Health Profile is repeated six months after intervention and two years after randomisation. Other cardiovascular events occurring during follow up, including stroke and hospital admission for unstable angina, are also recorded. In order to evaluate the cost effectiveness of the different treatment policies the duration of all hospital admissions, including admissions to high dependency units, and the details of all repeat coronary arteriograms and repeat intervention procedures are documented.

\section{Ethical considerations}

The trial protocol has received ethical approval from the hospital ethics committees of all participating centres. The nature of the trial is explained in detail to all patients who are required to sign a standardised consent form before randomisation. Patients are at liberty to withdraw from the study at any time, although they are encouraged to decline randomisation unless they are prepared to proceed with the assigned intervention procedure.

\section{Statistical considerations}

It is intended that at least 1000 patients will be entered in the trial, the primary consideration being a reliable comparison of the rate of new myocardial infarction or death. If in five years there is a $20 \%$ incidence of this combined end point in one group, a trial of this size would have $80 \%$ power to detect a one third reduction in the incidence in the other group at the $5 \%$ significance level. Since the five year fatality rate will probably be lower than $20 \%$, a trial of 1000 patients is likely to be too small to establish or refute that the treatments are equivalent for long term survival. However, 1000 patients will be sufficient to compare other end points such as symptoms, quality of life, exercise performance, and ejection fraction.

\section{Trial organisation}

The Randomised Intervention Treatment of Angina Trial comprises fourteen participating centres and a complex management structure including a steering committee of investigators, an executive committee, an ethics committee, and a statistical centre. The steering committee has overall responsibility for the 
conduct of the trial, but day to day management is supervised by the executive committee, which also monitors progress at each participating centre. The ethics committee is concerned with the welfare of the patients included in the trial, and is the only committee to which the comparative trial results will be available while the trial is in progress. The statistical centre is responsible for the randomisation of patients, and is maintaining the randomised trial data and the coronary arteriogram register. Each participating centre has employed a research assistant (usually an experienced nurse) to supervise the trial procedures and organise local data collection.

\section{Conclusion}

The protocol for the Randomised Intervention Treatment of Angina Trial has been described. The purpose of the study is to compare coronary angioplasty and coronary bypass surgery in suitable patients with coronary artery disease, and this can only reliably be achieved by a randomised clinical trial. Large multicentre clinical trials are difficult to conduct and require a multidisciplinary approach with cooperation across academic and clinical boundaries. Nevertheless, the Randomised Intervention Treatment of Angina Trial addresses an important issue and the results are likely to have major implications for the future management of patients with coronary artery disease. The results are also likely to influence allocation of resources for two expensive yet different methods of treatment.

The RITA Trial Steering Committee gratefully acknowledges additional financial support received from Advanced Cardiovascular Systems (USA), Medtronic (UK), and Schneider (UK).

\section{Appendix}

STEERING COMMITTEE OF INVESTIGATORS:

E Sowton (chairman), A K Yates (Guy's Hospital, London); C W Pumphrey, J R Pepper (St George's Hospital, London); P J B Hubner, R K Firmin (Groby Road Hospital, Leicester); R Balcon, P McGee (The London Chest Hospital, London); D C Cumberland, G H Smith (Northern General Hospital, Sheffield); K A A Fox, I M Breckenridge (University Hospital of Wales, Cardiff); N Brooks, $H$ Moussalli (Wythenshawe Hospital, Manchester); S M Cobbe, D J Wheatley (Royal Infirmary, Glas- gow); D S Dymond, S J Edmondson (St Bartholomews Hospital, London); D B O'Keefe, H O'Kane (Belfast City Hospital, Belfast); H C Miller, E W J Cameron (Royal Infirmary of Edinburgh, Edinburgh); M T Rothman, A J Wood (London Hospital, London); R $\mathbf{H}$ Swanton, $\mathrm{T}$ Treasure (Middlesex Hospital, London); D E Jewitt, J R W Keates (King's College Hospital, London)

\section{EXECUTIVE COMMITTEE:}

J R Hampton (chairman), E Sowton, S J Pocock, D G Julian, J Wallwork, R A Henderson

ETHICS COMMITTEE:

D A Chamberlain (chairman), M D Joy, J F Dark, S J Pocock

STATISTICAL CENTRE:

Clinical Trials Research Unit, Royal Free Hospital School of Medicine

\section{References}

1 The Veterans Administration coronary artery bypass surgery cooperative study group. Eleven-year survival in the Veterans Administration randomised trial of coronary bypass surgery for stable angina. $N$ Engl J Med 1984;311:1333-9.

2 European Coronary Surgery Study Group. Long-term results of prospective randomised study of coronary artery bypass surgery in stable angina pectoris. Lancet 1982;ii:1173-80.

3 CASS principal investigators and their associates. Coronary artery surgery study (CASS): a randomised trial of coronary artery bypass surgery. Survival data. Circulation 1983;68:939-50.

4 Detre K, Holubkov R, Kelsey S, et al. Percutaneous transluminal coronary angioplasty in 1985-1986 and 1977-1981. The National Heart, Lung, and Blood $\exists$ Institute Registry. N Engl J Med 1988;318:265-70.

5 Kent KM, Bentivoglio LG, Block PC, et al. Long-term efficacy of percutaneous transluminal coronary angioplasty (PTCA): report from the National Heart, 은 Lung, and Blood Institute PTCA Registry. Am J Cardiol 1984;53:27C-31C.

6 Cambeau L. Grading of angina pectoris. Circulation 1976;54:522-3.

7 Backett E, McEwen J, Hunt S. Report to the Social N Science Research Council. Health and quality of life. N 1981.

8 Sheffield LT. Graded exercise test (GXT) for ischaemic heart disease. A submaximal test to a target $\stackrel{\circ}{\subset}$ heart rate. In: Exercise testing and training of apparen- $\mathbb{D}$ tly healthy individuals. A handbook for physicians. Dallas: American Heart Association Committee on 7 Exercise, 1972:35-8. 